\title{
Comparison of sunscreens Containing Titanium Dioxide Alone Or In Association With Cocoa, Murumuru Or Cupuaçu Butters
}

\author{
Comparação de Protetores Solares Contendo Dióxido De Titânio Associado Ou Não A \\ Manteigas De Cacau, Murumuru Ou Cupuaçu
}

\author{
Andrea Marronato ${ }^{1}$, Tania Santos de Almeida ${ }^{2}$, Joana Portugal Mota ${ }^{2}$, Camila Areias de Oliveira ${ }^{1}$, \\ Catarina Rosado $^{2}$, Maria Valéria Robles Velasco ${ }^{1}$, André Rolim Baby ${ }^{1}$ \\ ${ }^{1}$ Faculdade de Ciências Farmacêuticas, Universidade de São Paulo, Av. Prof. Lineu Prestes, 580, Bloco 15, 05508-900, São Paulo, Brasil \\ ${ }^{2}$ CBios - Research Center for Biosciences and Health Technologies, U Lusófona, Campo Grande 376, 1749-024 Lisboa, Portugal \\ Email: andrerb@usp.br \\ Fontes de financiamento: FAPESP (Processo 10/10739-5)
}

\begin{abstract}
Ingredients of natural origin may represent alternatives for formulating sunscreens, without compromising their effectiveness. The literature has shown the antioxidant potential of compounds existing in murumuru (Astrocaryum murmuru), cupuaçu (Theobroma grandiflorum) and cocoa (Theobroma cacao) butters that recommends further investigation. The objectives of this research were: (1) to develop bioactive photoprotective formulations containing cocoa, murmuru or cupuaçu butters in association with a physical sunscreen (titanium dioxide (TiO2)) and (2) determine the possible photoprotective activity of butters and their interactions with the sunscreen. Cocoa, cupuaçu and murumuru butter were individually associated to $\mathrm{TiO} 2$ in $\mathrm{O} / \mathrm{W}$ emulsions. The anti-UVA and UVB efficacy in vitro was estimated

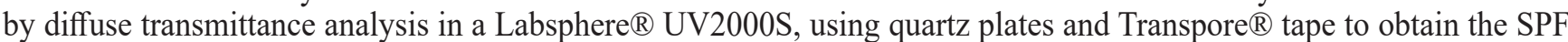
(sun protection factor) and critical wavelength. Photoprotective formulations containing titanium dioxide and bioactive butters were obtained using Aristoflex ${ }^{\circledR}$ AVC and triglycerides of caprylic capric acid (GTCC). The SPF of the samples containing only $\mathrm{TiO} 2$ and formulations containing cocoa, murumuru or cupuassu butters associated with $\mathrm{TiO} 2$ ranged from 4 to 5. The critical wavelength values for these formulations ranged from 383.0 to $386.7 \mathrm{~nm}$. The results indicated no increase in the SPF value for formulations containing vegetable butters. The critical wavelength values indicated their potential to absorb part of the UVA radiation.
\end{abstract}

Keywords: titanium dioxide, bioactive butters, Theobroma cacao, Theobroma grandiflorum, Astrocaryum murumuru, in vitro SPF

\section{Resumo}

Os ingredientes de origem natural podem representar alternativas para formulação de protetores solares, sem que haja comprometimento de sua eficácia. A literatura tem indicado potencial antioxidante de compostos existentes nas manteigas de murumuru (Astrocaryum murumuru), cupuaçu (Theobroma grandiflorum) e cacau (Theobroma cacao) que necessitam de maior investigação. Os objetivos desta pesquisa foram: (1) desenvolver fotoprotetores bioativos contendo manteigas de cacau (Theobroma cacao), murumuru (Astrocaryum murumuru) ou cupuaçu (Theobroma grandiflorum (Willd. Ex. Spring) Schum) em associação com filtro solar físico (dióxido de titânio) e (2) determinar a possível atividade fotoprotetora das manteigas e suas interações com filtro solar. As manteigas de cacau, cupuaçu e murumuru foram associadas, individualmente, ao dióxido de titânio em emulsões O/A. A eficácia anti-UVA e UVB in vitro foi estimada por meio da análise de transmitância difusa realizada em Labsphere ${ }^{\circledR}$ UV2000S utilizando placas de quartzo e fita Transpore ${ }^{\circledR}$ para obtenção do FPS (fator de proteção solar) e comprimento de onda crítico. Formulações fotoprotetoras contendo dióxido de titânio e manteigas bioativas foram obtidas utilizando-se Aristoflex ${ }^{\circledR}$ AVC e triglicérides do ácido cáprico cáprilico (GTCC). O FPS das amostras contendo apenas dióxido de titânio (TiO2) e das formulações contendo manteigas de cacau, murumuru ou cupuaçu associadas ao $\mathrm{TiO} 2$ variou entre 4 a 5. Os valores de comprimento de onda crítico para estas formulações foram no intervalo de 383,0 a 386,7 nm. Os resultados obtidos não indicaram incremento no valor de FPS para as formulações contendo manteigas vegetais. Os valores comprimento de onda crítico indicaram seu potencial para absorver parte da radiação UVA.

Palavras-chave: dióxido de titânio, manteigas bioativas, Theobroma cacao, Theobroma grandiflorum, Astrocaryum murumuru, FPS in vitro 


\section{Introduction}

Several studies have indicated the damages caused by exposure to ultraviolet (UV) radiation (1-5). Among the radiation emitted by the sun, UV radiation is the most energetic and most able to reach the Earth's surface. Currently, it is known that this radiation can be absorbed by biomolecules such as proteins, DNA and RNA. This absorption promotes cumulative metabolic and dose-dependent damages. These effects can lead to increased cytokines and inflammatory mediators, photosensitivity reactions, and, long-term, skin cancer (6). UV radiation can be divided into UVA (320 to 400 $\mathrm{nm}$ ), UVB (290 to $320 \mathrm{~nm}$ ) and UVC (100 to 290nm) $(5,7)$. The UVC radiation is the most energetic, but it is blocked by the Earth's ozone layer. UVA radiation is responsible for the photoaging process affecting components of the dermis and epidermis. It can also generate reactive oxygen species (ROS) that operate in many oxidation reactions, affecting nucleic acids, proteins, and lipid membranes. The damage caused by UVB radiation includes pyrimidine dimer formation, favouring changes in the DNA double helix, damaging replication and gene expression $(2,8)$

In this context, prevention of sun exposure and daily and adequate use of sunscreens plays a key strategy in preventing acute and chronic damage caused by UV radiation, including photo-carcinogenesis and photoaging $(5,9,10)$. Sunscreens must have characteristics to achieve adequate performance, including spreadability, pleasant sensory features, and no penetration through the stratum corneum, among others (10).

Despite the sunscreen market being on the rise, some factors still limit a higher adoption of use (1). Among them is their high cost, caused by the high value of their raw materials. Other reasons are related to the existence of controversial studies that show a possible allergenic potential or even toxicity caused by the use of sunscreens and possible degradation products, particularly in the case of organic filters (11-13). This has generated a trend to seek new products with higher sunscreen effectiveness, chemical stability, lower allergenic potential and lower cost.

In the past attempts to find alternative ingredients and photoprotective action for use in cosmetics, there was a significant increase in studies involving compounds of natural origin. The research strategy for new compounds involves potential antioxidant activity and/or the presence of chromophores in a molecule that exhibits activity in UV absorption $(1,9)$. The use of antioxidants such as flavonoids, such as , compounds commonly found in vegetables with the function to protect

\section{Introdução}

Diversos estudos têm indicado os danos provocados pela exposição à radiação ultravioleta (UV) (1-5). Dentre as radiações emitidas pelo sol, a mais energética, capaz de atingir a superfície terrestre é a UV. Atualmente, sabe-se que esta pode ser absorvida por biomoléculas como proteínas, DNA e RNA. Tal fato promove alterações metabólicas cumulativas e dose-dependentes. Estes efeitos podem gerar aumento de citocinas e mediadores inflamatórios, reações de fotossensibilidade e, em longo prazo, câncer de pele (6).

Pode-se dividir a radiação UV em UVA (320 a $400 \mathrm{~nm})$, UVB (290 a $320 \mathrm{~nm})$ e UVC (100 a 290nm) (5,7). A radiação UVC é a mais energética, porém é bloqueada pela camada de ozônio. A radiação UVA é responsável pelo processo de fotoenvelhecimento cutâneo, afetando componentes da derme e epiderme. Pode, ainda, gerar espécies reativas de oxigênio (EROs) que atuam em inúmeras reações de oxidação, afetando ácidos nucléicos, proteínas e membranas lipídicas. Os danos provocados pela radiação UVB incluem formação de dímeros de pirimidina que favorecem alterações na dupla hélice de DNA, prejudicando a replicação e expressão gênica $(2,8)$

Neste contexto, a prevenção à exposição solar e o uso diário e adequado de protetores solares exerce estratégia fundamental na prevenção contra danos agudos e crônicos causados pela radiação UV, entre eles fotocarcinogênese e fotoenvelhecimento $(5,9,10)$. Os protetores solares devem apresentar características para atingirem performance adequada, entre elas: espalhabilidade, sensorial agradável, além de não penetrar através do estrato córneo, dentre outras (10).

Apesar do mercado de protetores solares estar em ascensão, alguns fatores ainda são limitantes a um maior consumo (1). Entre eles estão o seu alto custo, ocasionado pelo alto valor de suas matérias-primas. Outras razões se relacionam à existência de estudos controversos que indicam possível potencial alergênico ou até mesmo tóxico, ocasionado pelo uso de filtros solares e possíveis produtos de degradação, principalmente no caso de filtros orgânicos (11-13). Este fato tem gerado tendência a buscar novos produtos com maior eficácia antissolar, estabilidade química, menor potencial alergênico e menor custo.

$\mathrm{Na}$ tentativa de encontrar ingredientes alternativos e com ação fotoprotetora para aplicação em cosméticos, ocorreu sensível aumento nos estudos envolvendo compostos de origem natural. A estratégia de investigação de novos compostos envolve potencial ação antioxidante e/ou presença de cromóforos em sua molécula que 
them against UV radiation, could provide synergistic and complementary role in sunscreens for improved sun protection $(8,14-17)$. Some studies have shown favourable evidence regarding the use of oils and plant extracts in formulations aimed at sun protection $(8,17,18)$. In view of the Brazilian biodiversity, some species, although already present in cosmetic use, require further studies of their potential photoprotective and antioxidant activities. Among these species:

\section{Cocoa (Theobroma cacao)}

Cocoa butter is extracted from the cacao fruit seed and has known applications in cosmetic creams, lotions and lip products, acting as an emollient with a silky touch and preventing transepidermal water loss. It is rich in fatty acids (palmitic, stearic and oleic) (19). OLIVEIRA et al (20) demonstrated the antioxidant activity of cocoa flavonoids. The literature also provides information on their use in food and topical use in cosmetic formulations. (21). Other published studies indicate a potential photoprotective activity of cocoa polyphenols (Santos, et al., 2010), and their ability to provide increased blood flow helps the skin restructure and improves the overall appearance of the skin $(14,21)$.

\section{Cupuaçu (Theobroma grandiflorum (Willd. Ex. Spring) Schum)}

The cupuaçu is an endemic tree of the Amazon whose fruits have a white pulp, and aroma and flavour similar to that of cocoa (19). The cupuaçu butter is obtained from the seed of the fruit, and its composition includes fatty acids and long chain phytosterols. Its known cosmetic uses include hair and skin products due to its moisturizing and emollient properties (19). The cupuaçu phenolic content may indicate antioxidant activity and potential as a sunscreen (22)

\section{Murumuru (Astrocaryum murumuru)}

The murumuru palm is very common in the Amazon Basin and produces reddish fruits made of almond and pulp. Murumuru butter is obtained from the seeds and has cosmetic application as an emollient. It is rich in lauric, myristic and oleic acids. It also contains phytosterols, the most important being $\beta$-sitosterol $(19,23)$. The objectives of this research study were: (1) developing bioactive photoprotective containing cocoa, murmuru or cupuaçu butters in association with a physical sunscreen (titanium dioxide, $\mathrm{TiO}_{2}$ ) and (2) determine the possible photoprotective activity of these butters and their interactions with the sunscreen. exibiriam ação na absorção da radiação UV $(1,9)$. O uso de antioxidantes, como flavonoides, por exemplo, compostos comumente encontrados em vegetais com a função de protegê-los contra radiação UV, poderia apresentar papel sinérgico e complementar os filtros solares, atuando na proteção solar $(8,14-17)$. Alguns estudos já demonstraram evidências favoráveis com relação ao uso de óleos e extratos vegetais em formulações voltadas à proteção solar $(8,17,18)$.

Tendo em vista a Biodiversidade Brasileira, algumas espécies, apesar de já apresentarem utilização em cosméticos, necessitam de estudos complementares sobre suas potenciais atividades antioxidantes e fotoprotetoras. Dentre estas espécies:

\section{Cacau (Theobroma cacao)}

A manteiga de cacau é extraída da semente do fruto do cacaueiro e possui aplicações conhecidas em cosméticos em cremes, loções e produtos labiais, atuando como emoliente de toque sedoso e evitando perda transepidérmica de água. É rica em ácidos graxos (palmítico, esteárico e oléico) (19). OLIVEIRA e colaboradores (20) demonstraram a atividade antioxidante dos flavonoides do cacau. A literatura também traz informações sobre seu consumo em alimentos e seu uso tópico veiculado em formulações cosméticas (21). A literatura possui estudos que indicaram uma potencial atividade fotoprotetora dos polifenóis do cacau (16), além da capacidade de proporcionar um aumento do fluxo sanguíneo que contribui na reestruturação cutânea e melhoria da aparência geral da pele $(14,21)$.

\section{Cupuaçu (Theobroma grandiflorum (Willd. Ex. Spring)} Schum)

O cupuaçuzeiro é uma árvore endêmica da Amazônia cujos frutos apresentam polpa branca, aromática e sabor semelhante ao do cacau (Theobroma cacao) (19). A manteiga de cupuaçu é obtida a partir da semente do fruto e apresenta em sua composição, ácidos graxos de cadeia longa e fitosteróis. Seu uso cosmético conhecido inclui produtos capilares e para pele, graças às suas propriedades hidratantes e emolientes (19). O conteúdo de fenóis do cupuaçu pode indicar atividade antioxidante e potencial como protetor solar (22)

\section{Murumuru (Astrocaryum murumuru)}

A palmeira de murumuru é muito comum na Bacia Amazônica e dá origem a frutos avermelhados constituídos de amêndoa e polpa. A manteiga de murumuru é obtida a partir das sementes e tem aplicação cosmética como emoliente. É rica em ácidos láurico, mirístico e 


\section{Materials and Methods}

\section{Materials}

The formulation contained the following ingredients: Caprylic/capric triglyceride, Crodamol ${ }^{\circledR}$ GTCC, Croda; Ammonium acryloyldimethyltaurate / VP copolymer, Aristoflex ${ }^{\circledR}$ AVC, Clariant S.A; BHT; EDTA disodium; Phenoxyethanol (and) methylparaben (and) ethylparaben (and) propylparaben (and) butylparaben (and) isobutylparaben, Phenova ${ }^{\circledR}$, Croda; Triethanolamine; Water; Titanium Dioxide (and) Caprylic/ Capric Triglyceride (and) Tri-PPG-3 Myristyl Ether Citrate (and) Sorbitan Isostearate (and) Polyglyceryl-3 Polyricinoleate (and) Manganese Oxide), Optisol ${ }^{\circledR}$ TD50, Croda; Murumuru butter (Astrocaryum murumuru), Crodamazon ${ }^{\circledR}$ Murumuru, Croda; Cupuaçu butter (Theobroma grandiflorum), Crodamazon ${ }^{\circledR}$ Cupuaçu, Croda; Cocoa butter (Theobroma cacao), Crodamazon ${ }^{\circledR}$ Cacau, Croda

Analytical measurements were made with a Labsphere UV 2000S UV transmittance analyzer and viscosity was measured with a Visco Star-R viscometer. oléico. Possui também fitosteróis sendo o de maior importância o $\beta$-sitosterol $(19,23)$.

Esta pesquisa teve como objetivos: (1) Desenvolver fotoprotetores bioativos contendo manteigas de cacau, murumuru ou cupuaçu em associação com filtro solar físico (dióxido de titânio) e (2) Determinar a possível atividade fotoprotetora das manteigas de cacau, murumuru e cupuaçu e sua interação com filtro solar.

\section{Material e Métodos}

\section{Materiais}

Os ingredientes da formulação estão apresentados a seguir: Triglicérides do ácido cáprico caprílico (caprylic) capric triglyceride), Crodamol ${ }^{\circledR}$ GTCC, Croda; Polímero acriloildimetiltaurato de amônio/copolímero de vinilpirrolidona (ammonium acryloyldimethyltaurate / VP copolymer), Aristoflex ${ }^{\circledR}$ AVC, Clariant S.A; BHT; EDTA dissódico; Phenoxyethanol (and) methylparaben (and) ethylparaben (and) propylparaben (and) butylpa-

Table 1/ Tabela 1 - Composition of the tested formulations $(\% \mathrm{w} / \mathrm{w})$ Composição das formulações testadas $(\% \mathrm{p} / \mathrm{p})$

Aristoflex ${ }^{\circledR}$ AVC (Ammonium acryloyldimethyltaurate / VP copolymer); GTCC (Caprylic/capric triglyceride); EDTA disodium; BHT (di-terc-butil metil phenol), Phenova ${ }^{\circledR}$ (Phenoxyethanol (and) methylparaben (and) ethylparaben (and) propylparaben (and) butylparaben (and) isobutylparaben). A to E (formulations)

Aristoflex ${ }^{\circledR}$ AVC (acriloildimetiltaurato de amônio/copolímero de vinilpirrolidona); GTCC (triglicérides do ácido cáprico caprílico); EDTA dissódico; BHT (di-terc-butil metil fenol), Phenova ${ }^{\circledR}$ (Fenoxietanol, metiparabeno, etilparabeno, propilparabeno, butilparabeno e isobutilparabeno). A a E (formulações).

\begin{tabular}{lccccc}
\hline $\begin{array}{l}\text { Components } \\
\text { Componentes }\end{array}$ & \multicolumn{5}{c}{ Formulations } \\
& A & B & C & D & E \\
Aristoflex ${ }^{(8)} \mathrm{AVC}$ & $0,5 \%$ & $1,0 \%$ & $2,0 \%$ & $3,0 \%$ & $2,0 \%$ \\
GTCC & $5,0 \%$ & $5,0 \%$ & $5,0 \%$ & $5,0 \%$ & $10,0 \%$ \\
EDTA & $0,1 \%$ & $0,1 \%$ & $0,1 \%$ & $0,1 \%$ & $0,1 \%$ \\
BHT & $0,5 \%$ & $0,5 \%$ & $0,5 \%$ & $0,5 \%$ & $0,5 \%$ \\
Phenova & $0,5 \%$ & $0,5 \%$ & $0,5 \%$ & $0,5 \%$ & $0,5 \%$ \\
Triethanolamine & q.s.pH $6,0-7,0$ & q.s.pH $6,0-7,0$ & q.s.pH $6,0-7,0$ & q.s.pH $6,0-7,0$ & q.s.pH $6,0-7,0$ \\
/Triethanolamine & & & & & q.s. \\
Water/Água & q.s. & q.s. & & & q.s. \\
\hline
\end{tabular}


Table 2/ Tabela 2 - Quantitative composition and functions of each component of the Blank Formulation $(\% \mathrm{w} / \mathrm{w})$

Fórmula quantitativa e funções de cada componente da Formulação Branco ( $\% \mathrm{p} / \mathrm{p})$

Aristoflex ${ }^{\circledR}$ AVC (Ammonium acryloyldimethyltaurate / VP copolymer); GTCC (Caprylic/capric triglyceride); EDTA disodium; BHT (di-terc-butil metil phenol), Phenova ${ }^{\circledR}$ (Phenoxyethanol (and) methylparaben (and) ethylparaben (and) propylparaben (and) butylparaben (and) isobutylparaben). Aristoflex ${ }^{\circledR}$ AVC (acriloildimetiltaurato de amônio/copolímero de vinilpirrolidona); GTCC (triglicérides do ácido cáprico caprílico); EDTA dissódico; BHT (di-terc-butil metil fenol), Phenova

(Fenoxietanol, metiparabeno, etilparabeno, propilparabeno, butilparabeno e isobutilparabeno).

\begin{tabular}{lcc}
\hline $\begin{array}{l}\text { Components } \\
\text { Componentes }\end{array}$ & $\begin{array}{c}\text { Function } \\
\text { Função }\end{array}$ & $\begin{array}{c}\text { Concentration (\%) } \\
\text { Concentração (\%) }\end{array}$ \\
\hline \multirow{2}{*}{ Aristoflex ${ }^{\circledR}$ AVC } & Surfactant/thickening & \\
GTCC & Tensoativo/ Espessante & 2.0 \\
EDTA & Emollient/Emoliente & 5.0 \\
BHT & Chelator/Quelante & 0.1 \\
Phenova & Antioxidant/Antioxidante & 0.5 \\
Triethanolamine /Triethanolamine & Preservative/Conservante & 0.5 \\
Water/Água & pH adjusting/Corrector de pH & 1.0 \\
\hline
\end{tabular}

\section{Preparation and emulsion tests}

Formulations with different concentrations of Aristoflex ${ }^{\circledR}$ AVC and caprylic/ capric acid triglycerides were developed without the butters or the titanium dioxide suspension in order to obtain a basic formulation with suitable characteristics. Table 1 shows the components of the initial formulation and the concentration range of the components Aristoflex ${ }^{\circledR}$ AVC and triglycerides of caprylic /capric acid.

Table 2 shows the qualitative and quantitative content selected for the basic formulation, referred hereafter as the Blank. The preparation was performed by weighing the components of the aqueous phase (EDTA and water) and heating to $40^{\circ} \mathrm{C}$. The ingredients of the oil phase (caprylic capric acid triglycerides, Aristoflex ${ }^{\circledR}$ AVC and BHT), were then weighed, homogenized, and heated to $40-45^{\circ} \mathrm{C}$. After pouring the aqueous phase on the oil (reversed phase), using manual agitation an emulsion was formed with a gel - cream appearance.

Optisol ${ }^{\circledR}$ TD -50 was added in the oil phase of the formulation at concentrations of $5.0 \%(\mathrm{w} / \mathrm{w})$ and $10.0 \%$ $(\mathrm{w} / \mathrm{w})$ for subsequent evaluation and selection of the optimal concentration to be used in the study. This new formulation was named Standard. Table 3 describes the concentration of each component.

The concentration of vegetable butters defined for inclusion within formulations was $5.0 \%(\mathrm{w} / \mathrm{w})$, based on the information from the supplier (Croda). Butters were melted at a temperature between $35^{\circ}-40^{\circ} \mathrm{C}$ and incor- raben (and) isobutylparaben, Phenova ${ }^{\circledR}$, Croda; Trietanolamina (Triethanolamine); Água (aqua) - veículo; Dióxido de titânio, triglicérides do ácido cáprico/ caprílico, tri- PPG-3 miristil éter citrato, isostearato de sorbitan, poligliceril-3 poliricinoleato e óxido de manganês (Titanium Dioxide (and) Caprylic/Capric Triglyceride (and) Tri-PPG-3 Myristyl Ether Citrate (and) Sorbitan Isostearate (and) Polyglyceryl-3 Polyricinoleate (and) Manganese Oxide), Optisol ${ }^{\circledR}$ TD-50, Croda - suspensão de $50 \%$ dióxido de titânio (filtro físico de amplo espectro); Manteiga de murumuru (Astrocaryum murumuru), Crodamazon ${ }^{\circledR}$ Murumuru, Croda; Manteiga de cupuaçu (Theobroma grandiflorum), Crodamazon ${ }^{\circledR}$ Cupuaçu, Croda; Manteiga de cacau (Theobroma cacao), Crodamazon ${ }^{\circledR}$ Cacau, Croda.

Medições analíticas de transmitância foram efectuadas com um Labsphere UV 2000S UV e a viscosidade foi medida com um Visco Star-R viscometer.

\section{Preparação e testes de emulsões}

Formulações com diferentes concentrações de Aristoflex ${ }^{\circledR}$ AVC e triglicérides do ácido cáprico caprílico foram desenvolvidas sem as manteigas ou a suspensão de dióxido de titânio com a finalidade de obter formulação base com características adequadas. A Tabela 1 apresenta os componentes da formulação inicial e o intervalo de concentração dos componentes Aristoflex ${ }^{\circledR}$ AVC e triglicérides do ácido cáprico caprílico.

A Tabela 2 apresenta o conteúdo qualitativo e quantitati- 
Table 3/ Tabela 3 - Quantitative composition and functions of each component Standard Formulation (\% w/w) Fórmula quantitativa e funções de cada componente da Formulação Padrão (\% p/p)

Equal to Blank formulation (Table 2) with the addition of Optisol® TD-50 (Titanium Dioxide (and) Caprylic/ Capric Triglyceride (and) Polyglyceryl-3 Polyricinoleate (and) Di-PPG-3 Myristyl Ether Adipate (and) Sorbitan Isostearate (and) Manganese Oxide

Igual à formulação Branco (Tabela 2) com a adição de Optisol® (Dióxido de titânio, triglicérides do ácido cáprico/ caprílico, tri- PPG-3 miristil éter citrato, isostearato de sorbitan, poligliceril-3 poliricinoleato e óxido de manganês).

\begin{tabular}{lcc}
\hline $\begin{array}{l}\text { Components } \\
\text { Componentes }\end{array}$ & $\begin{array}{c}\text { Function } \\
\text { Função }\end{array}$ & $\begin{array}{c}\text { Concentration (\%) } \\
\text { Concentração (\%) }\end{array}$ \\
\hline Aristoflex ${ }^{\circledR}$ AVC & Surfactant/thickening & 2,0 \\
GTCC & Tensoativo/ Espessante & \\
Optisol TD-50 & Emollient/ Emoliente & 5,0 \\
EDTA & Chelator/Quelante & 5,0 \\
BHT & Antioxidant/ Antioxidante & 0,1 \\
Phenova ${ }^{\circledR}$ & Preservative/Conservante & 0,5 \\
Triethanolamine/ & pH adjusting/ Corretor de pH & 0,5 \\
Trietanolamina & Vehicle/ Veículo & 1,0 \\
Water/Água & & q.s.p./qs \\
\hline
\end{tabular}

Table 4/ Tabela 4 - Quantitative Formula and functions of each component of Formulation $\mathrm{F}$ [Murumuru butter (Astrocaryum murumuru ) ] in \% w/w.

Fórmula Quantitativa e funções de cada componente da Formulação F [Manteiga de Murumuru (Astrocaryum murumuru)] em\% p/p.

\begin{tabular}{|c|c|c|}
\hline $\begin{array}{l}\text { Components } \\
\text { Componentes }\end{array}$ & $\begin{array}{l}\text { Function } \\
\text { Função }\end{array}$ & $\begin{array}{c}\text { Concentration } \\
\text { (\%) } \\
\text { Concentração } \\
(\%)\end{array}$ \\
\hline Aristoflex ${ }^{\circledR}$ AVC & $\begin{array}{l}\text { Surfactant/thickening } \\
\text { Tensoativo/ Espessante }\end{array}$ & 2,0 \\
\hline $\begin{array}{l}\text { GTCC } \\
\text { Titanium dioxide/ }\end{array}$ & $\begin{array}{l}\text { Emollient/ Emoliente } \\
\text { Physical sunscreen/ }\end{array}$ & 5,0 \\
\hline Dióxido de Titânio & $\begin{array}{c}\text { Filtro Solar Físico } \\
\text { Emollient with a potentential } \\
\text { photoprotective activity }\end{array}$ & 5,0 \\
\hline Manteiga de murumuru & $\begin{array}{l}\text { Emoliente e possível auxiliar } \\
\text { na atividade fotoprotetora. }\end{array}$ & 5,0 \\
\hline EDTA & Chelator/Quelante & 0,1 \\
\hline BHT & Antioxidant/ Antioxidante & 0,5 \\
\hline Phenova & Preservative/Conservante & 0,5 \\
\hline Triethanolamine & $\mathrm{pH}$ adjusting/ Corretor de $\mathrm{pH}$ & 1,0 \\
\hline Water/Àgua & Vehicle/Veículo & $\mathrm{q}, \mathrm{s}$ \\
\hline
\end{tabular}


porated into the formulation. Formulations containing murumuru butter, cocoa and cupuassu were labeled as $\mathrm{F}, \mathrm{G}$ and $\mathrm{H}$, respectively. The qualitative contents and concentrations of each component are described in Tables 4,5 and 6 .

\section{In vitro determination of SPF and UVA index}

In vitro efficacy was estimated by the diffuse transmittance analysis for Labsphere ${ }^{\circledR}$ UV 2000S equipment to obtain UVA index and SPF (Labsphere ${ }^{\circledR}$, Technical Note). Samples were analyzed on plates of 50 x $50 \mathrm{~mm}$ quartz with Transpore ${ }^{\circledR}$ tape. Each plate contained 50 mg of sample, and measurements were made in duplicate at nine locations on the plate. The anti- UVA efficacy was determined from the analysis of the critical wavelength $(\lambda c)$ featuring the preparation and broad absorption spectrum (24). The coefficients of variation of these variables were also taken into account in the analysis.

\section{Statistical analysis}

The results of the SPF values and estimated UVA index for formulations containing the butters murumuru (Astrocaryum murumuru), cupuacu (Theobroma grandiflorum), or cacao (Theobroma cacao) were compared with the standard formulations by analysis of variance (ANOVA). The confidence interval was $95 \%$ and statistically significant differences were those with $\mathrm{p}<0.05$. vo selecionado para a Formulação Branca. O preparo foi realizado com a pesagem dos componentes da fase aquosa (EDTA e água) e seu aquecimento até $40^{\circ} \mathrm{C}$. Em seguida, procedeu-se com a pesagem dos ingredientes da fase oleosa (triglicérides do ácido cáprico caprílico, Aristoflex ${ }^{\circledR}$ AVC e BHT), os quais foram homogeneizados e aquecidos até $40-45^{\circ} \mathrm{C}$. Após verter a fase aquosa sobre a oleosa (inversão de fases), sob agitação manual, formou-se emulsão com aspecto de gel-creme.

O Optisol ${ }^{\circledR}$ TD-50 foi adicionado na fase oleosa da formulação nas concentrações de 5,0\% (p/p) e 10,0\% (p/p) para posterior avaliação de melhores características e seleção da concentração a ser utilizada no estudo. Esta nova formulação foi nomeada Padrão. A Tabela 3 descreve a concentração de cada componente utilizado.

A concentração de manteigas vegetais definida para a mistura nas formulações foi $5,0 \%(\mathrm{p} / \mathrm{p})$, com base das informações do fornecedor (Croda). As manteigas foram fundidas em temperatura entre $35^{\circ}-40^{\circ} \mathrm{C}$ e incorporadas à formulação. As formulações contendo manteiga de murumuru, cacau e cupuaçu foram denominadas respectivamente $\mathbf{F}, \mathbf{G}$ e $\mathbf{H}$ e apresenta seus conteúdos qualitativos e concentrações de cada componente descritos nas Tabelas 4, 5 e 6.

Table 5/ Tabela 5 - Quantitative Formula and functions of each component of Formulation G [(Cocoa butter (Theobroma cacao) in \% w/w.

Fórmula Quantitativa e funções de cada componente da Formulação G [(Manteiga de Cacau (Theobroma cacao)] em\% p/p.

\begin{tabular}{lcc}
\hline $\begin{array}{l}\text { Components/ } \\
\text { Componentes }\end{array}$ & Function/Função & $\begin{array}{c}\text { Concentration } \\
\text { (\%)/ } \\
\text { Concentração (\%) }\end{array}$ \\
\hline Aristoflex ${ }^{\circledR}$ AVC & Surfactant/thickening & 2.0 \\
GTCC & Tensoativo/ Espessante & 5.0 \\
Titanium dioxide & Emollient/Emoliente & 5,0 \\
& Physical sunscreen/ Filtro Solar Físico & \\
Emollient with a potentential photoprotective activity & \\
EDTA & Emoliente e possível auxiliar na atividade & 5,0 \\
BHT & fotoprotetora & 0,1 \\
Phenova & Chelator/ Quelante & 0,5 \\
Triethanolamine & Antioxidant/ Antioxidante & 0,5 \\
Water/ Àgua & Preservative/ Conservante & 1,0 \\
\hline
\end{tabular}




\section{Results}

Among the tested formulations, formulation $\mathrm{C}$ presented the best characteristics (according to Table 1), in which the concentrations of Aristoflex ${ }^{\circledR}$ AVC and caprylic capric acid triglyceride were respectively 2.0 and $5.0 \% \mathrm{w} / \mathrm{w}$. This emulsion showed a gel-cream consistency and typical odor, with adequate spreadability, texture and a pleasant (non-sticky) sensory feel.

The titanium dioxide concentration defined for the Standard formulation was 5.0\%. The Standard formulation characteristics were a rosy cream with an odour characteristic to Optsol ${ }^{\circledR}$ TD-50. The formulations F, G and $\mathrm{H}$ were pink in colour with odours typical of each applied butter as shown in Table 8 and in Figure 1.

Table 8 summarizes the in vitro efficacy results evaluated in quartz plates Transpore ${ }^{\circledR}$ tape. Figure 2 shows their respective spectral profiles.

The results of the analysis of variance for SPF and the Critical Lambda results by Tukey method are described in Tables 9, 10 and 11 and Figures 3, 4, 5 and 6.

Statistical results for the comparison of Standard formulation with the other formulations by the Tukey method showed significant differences between the SPF values for the Standard and Blank samples. However, results showed no difference between them in respect to critical Lambda values. Samples F, G and H showed SPF values and critical Lambda statistically identical to the Standard formulation.

\section{Determinação in vitro do FPS e UVA}

A eficácia UVA e UVB in vitro foi estimadas por meio da análise de transmitância difusa realizada em equipamento Labsphere ${ }^{\circledR}$ UV 2000S para obtenção de índice UVA e FPS (Labsphere ${ }^{\circledR}$, Technical Note). As amostras foram analisadas em placas de $50 \times 50 \mathrm{~mm}$ de quartzo com fita Transpore ${ }^{\circledR}$. Cada placa continha $50 \mathrm{mg}$ de amostra e as medições foram feitas em duplicata, em nove localizações da placa. A eficácia anti-UVA foi determinada a partir da análise do comprimento de onda crítico $(\lambda c)$ que caracteriza a preparação como de amplo espectro de absorção (24). Os coeficientes de variação destas variáveis também foram tidos em conta na análise.

\section{Análise estatística}

Os resultados dos valores de FPS e índice UVA estimados das formulações contendo manteigas de murumuru (Astrocaryum murumuru), cupuaçu (Theobroma grandiflorum) ou cacau (Theobroma cacao) foram comparados com os das formulações Padrão por meio de análise de variância (ANOVA). O intervalo de confiança considerado foi $95 \%$ e as diferenças estatisticamente significativas foram aquelas com $p<0,05$.

Table 6/ Tabela 6 - Quantitative Formula and functions of each component of Formulation /H [( Cupuaçu Butter (Theobroma grandiflorum) in \% w/w.

Fórmula Quantitativa e funções de cada componente da Formulação H [(Manteiga de Cupuaçu (Theobroma grandiflorum)) em $\% \mathrm{p} / \mathrm{p}$.

\begin{tabular}{lcc}
\hline Components & Function & Concentration (\%) \\
\hline Aristoflex ${ }^{\circledR}$ AVC & Surfactant/thickening & 2,0 \\
GTCC & Tensoativo/ Espessante & 5,0 \\
Titanium dioxide/ & Emollient Emoliente & \\
Dióxido de titânio & Physical sunscreen Filtro Solar Físico & 5,0 \\
& Emollient with a potentential photoprotective & \\
& activity & \\
Cupuaçu butter/ & Emoliente e possível auxiliar na atividade & \\
Manteiga de Cupuaçu & fotoprotetora & 5,0 \\
EDTA & Chelator/ Quelante & 0,1 \\
BHT & Antioxidant/ Antioxidante & 0,5 \\
Phenova & Preservative/Conservante & 0,5 \\
Trietanolamin/Triethanolamine & pH adjusting/corretor de pH & 1,0 \\
Water/ Àgua & Vehicle/Veículo & q,s.p \\
\hline
\end{tabular}


Table 7/ Tabela 7 - Organoleptic characteristics of the prepared formulations.

Características organolépticas das formulações preparadas.

\begin{tabular}{|c|c|c|c|}
\hline Formulation/ Formulação & $\begin{array}{l}\text { Aspect/ } \\
\text { Aspecto }\end{array}$ & $\begin{array}{c}\text { Colour/ } \\
\text { Cor }\end{array}$ & Odour/Odor \\
\hline Blank/ Branco & \multirow{5}{*}{ Gel- cream } & $\begin{array}{l}\text { White/ } \\
\text { Branco }\end{array}$ & $\begin{array}{l}\text { Typical and soft/ Suave e } \\
\text { característico }\end{array}$ \\
\hline Standard/ Padrão & & \multirow{4}{*}{$\begin{array}{l}\text { Rosy/ } \\
\text { Róseo }\end{array}$} & $\begin{array}{l}\text { Typical to Optisol }{ }^{\circledR} \text { TD-50/ } \\
\text { Característico de Optisol }{ }^{\circledR} \text { TD- } \\
50\end{array}$ \\
\hline $\begin{array}{l}\text { F (murumuru butter)/ } \\
\text { F (manteiga de murumuru) }\end{array}$ & & & $\begin{array}{l}\text { Typical to murumuru butter/ } \\
\text { Característico de manteiga de } \\
\text { murumuru }\end{array}$ \\
\hline $\begin{array}{l}\text { G (Cocoa Butter)/ G (manteiga de } \\
\text { cacau) }\end{array}$ & & & $\begin{array}{l}\text { Typical to cocoa butter/ } \\
\text { Característico de manteiga de } \\
\text { cacau }\end{array}$ \\
\hline $\begin{array}{l}\text { H (cupuaçu butter)/ H (manteiga de } \\
\text { cupuaçu) }\end{array}$ & & & $\begin{array}{l}\text { Typical to cupuaçu butter/ } \\
\text { Característico manteiga de } \\
\text { cupuaçu }\end{array}$ \\
\hline
\end{tabular}

\section{Discussion}

It was possible to develop photoprotective formulations containing $2.0 \%$ Aristoflex ${ }^{\circledR}$ AVC and $5.0 \%$ Caprylic/ Capric triglyceride (GTCC). No conventional surfactants where added to the formulation. This was possible due to the presence Aristoflex ${ }^{\circledR}$ AVC. Analysing its chemical structure, we found that each monomer of this polymer has $\mathrm{SO}^{3-} \mathrm{NH}^{4+}$ groups with polar feature. The remaining chain has nonpolar regions, facilitating its incorporation in the formulation in the oil phase. The application of GTCC emollient facilitated the dispersion of the active ingredients. The titanium dioxide suspension already contained this emollient among its components.

\section{Resultados}

Dentre as formulações testadas, a que apresentou melhores características foi a $\mathbf{C}$ (de acordo com a Tabela 1), na qual as concentrações de Aristoflex ${ }^{\circledR}$ AVC e triglicérides do ácido cáprico caprílico foram, respectivamente, 2,0 e 5,0\% p/p. Esta emulsão apresentou caráter de gel creme de coloração branca e odor característico, com adequada espalhabilidade, textura e sensorial agradável (não pegajoso).

A concentração de dióxido de titânio definida para a formulação Padrão foi 5,0\%. As características da Formulação Padrão foram de creme róseo com odor característico do Optsol ${ }^{\circledR}$ TD-50. As formulações $\mathbf{F}, \mathbf{G}$ e $\mathbf{H}$ apresentaram-se rosadas com odores característicos de cada manteiga aplicada como apresentado na Tabela 7 e podem ser visualizadas na Figura 1.

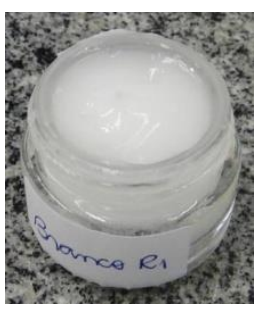

Blank/Branco

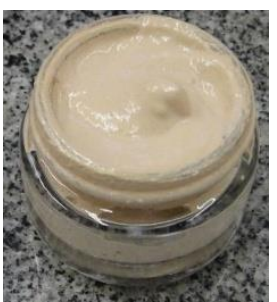

Standard/Padrão

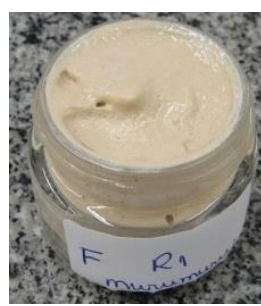

$\mathbf{F}$

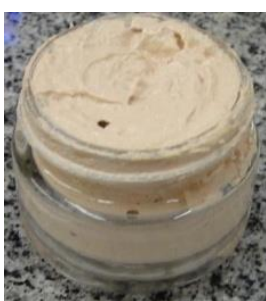

G

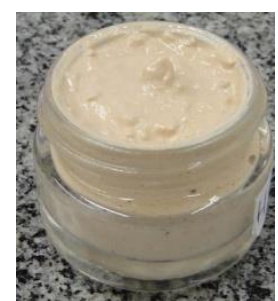

H

Figure 1/ Figura 1 - Photos of Blank formulations, Standard, $\mathrm{F}, \mathrm{G}$ and $\mathrm{H}$

Fotos das formulações Branco, Padrão, F, G e H 
The choice of these components for the formulation development allowed us to obtain a preparation with several advantageous features. Among them, the pleasant and not sticky (fatty) feel, a feeling of freshness as well as a good spreadability. However, this colour feature becomes advantageous in facial products as it could have multifunctional nature (possible correction of facial imperfections).

The SPF results were obtained through analysis using Transpore ${ }^{\circledR}$ tape and a Labsphere ${ }^{\circledR}$ UV2000S UV transmittance detector Table 8). This analysis methology offers low cost, easy handling, and is suitable for samples which do not have excess oil or alcohol as a carrier. The SPF values for the Blank formulation were significantly lower compared with the values of the other formulations. The formulations $\mathrm{F}, \mathrm{G}$ and $\mathrm{H}$ showed SPF values similar to those obtained from the Standard formulation containing only titanium dioxide. In these cases, the SPF values varied between 4 and 5. Thus, the vegetable butters showed no increase in the SPF of the formulations at the concentrations used, according to the results obtained by this analysis.
A Tabela 8 sumariza os resultados obtidos nos testes de eficácia in vitro das amostras avaliadas em placas de quartzo com fita Transpore ${ }^{\circledR}$ e a Figura 2 ilustra seus perfis espectrais.

Os resultados da Análise de variância para os resultados de FPS e Lambda crítico pelo método de Tukey estão descritos nas Tabelas 9, 10 e 11 e Figuras 3, 4, 5 e 6.

Os resultados estatísticos da comparação da formulação Padrão com as demais formulações pelo método de Tukey demonstraram diferenças significativas entre as amostras Padrão e Branco para valores de FPS. Porém, não demonstraram diferenças entre as mesmas em relação aos valores de Lambda crítico. As amostras F, G e $\mathrm{H}$ apresentaram valores de FPS e Lambda crítico estatisticamente iguais ao da formulação Padrão.
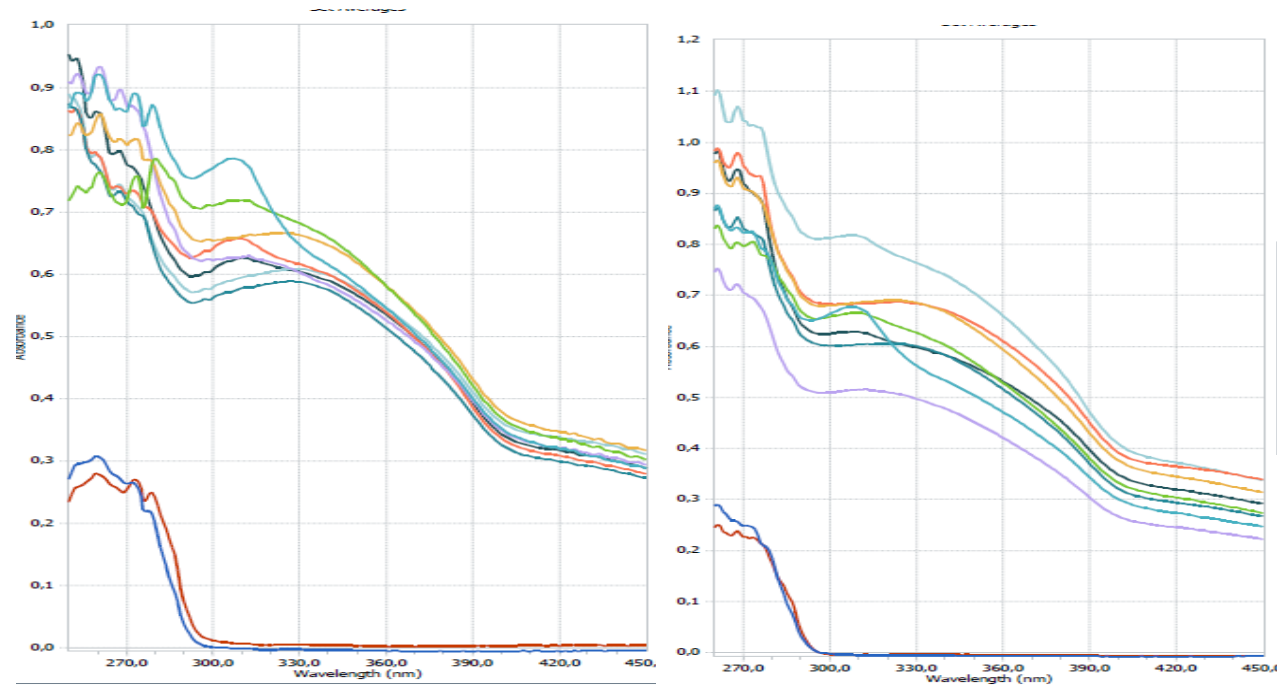

Legend/ Legenda
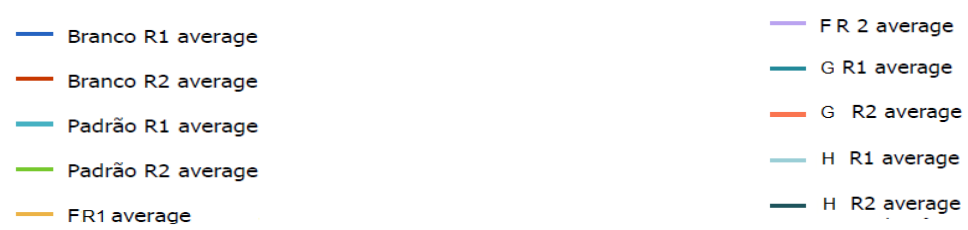

Figure 2/ Figura 2 - In vitro efficacy evaluated for the samples in quartz plates Transpore ${ }^{\circledR}$

Eficácia in vitro das amostras avalidas em placas de quartzo com Transpore ${ }^{\circledR}$ 
Table 8/ Tabela 8 - Results of the in vitro photoprotection efficacy test for the samples in quartz plates covered with Transpore ${ }^{\circledR}$.

Resultados do teste de eficácia de fotoprotecção in vitro para as amostras em placas de quartzo coberto com Transpore ${ }^{\circledR}$.

Grey cells have CV values higher than $20 \%$, not ideal for consideration; SPF (sun protection factor ; $\lambda \mathrm{c}$ (critical wavelength) ; CV ( coefficient of variation)

Células em cinza apresentam valores de COV maiores 20\%, não ideais para consideração; FPS (fator de proteção solar; $\lambda c$ (comprimento de onda crítico); COV (coeficiente de variação)

\begin{tabular}{|c|c|c|c|c|c|c|c|c|}
\hline \multirow[t]{2}{*}{$\begin{array}{l}\text { Sample } \\
\text { Amostra }\end{array}$} & \multicolumn{4}{|c|}{$\begin{array}{l}\text { Measurement } 1 \\
\text { Medição } 1\end{array}$} & \multicolumn{4}{|c|}{$\begin{array}{c}\text { Measurement } 2 \\
\text { Medição } 2\end{array}$} \\
\hline & $\begin{array}{l}\text { SPF } \\
\text { FPS }\end{array}$ & $\begin{array}{l}\mathrm{VC} \text { of } \\
\text { SPF(\%) } \\
\text { COV de } \\
\text { FPS }\end{array}$ & $\begin{array}{l}\text { Critical } \\
\text { wavelength } \\
(\lambda \mathrm{c}, \mathbf{n m}) \\
\text { CDO } \\
\text { crítico }\end{array}$ & $\begin{array}{l}\mathrm{VC} \text { of } \\
\lambda \mathrm{c} \\
(\%) \\
\mathrm{COV} \\
\text { de } \lambda \mathrm{c}\end{array}$ & $\begin{array}{l}\text { SPF } \\
\text { FPS }\end{array}$ & $\begin{array}{l}\text { VC of } \\
\text { SPF(\%) } \\
\text { COV de } \\
\text { FPS }\end{array}$ & $\begin{array}{l}\text { Critical } \\
\text { wavelength } \\
(\lambda \mathrm{c}, \mathrm{nm}) \\
\mathrm{CDO} \\
\text { crítico }\end{array}$ & $\begin{array}{l}\text { VC of } \\
\lambda c \\
(\%) \\
\text { COV } \\
\text { de } \lambda \mathrm{c}\end{array}$ \\
\hline $\begin{array}{l}\text { Blank R1 } \\
\text { Branco R1 }\end{array}$ & $\begin{array}{c}0,99 \pm \\
0,01\end{array}$ & 0,66 & 372,33 & 11,87 & $\begin{array}{c}0,99 \pm \\
0,01\end{array}$ & 0,67 & 393,44 & 0,51 \\
\hline $\begin{array}{l}\text { Blank R2 } \\
\text { Branco R2 }\end{array}$ & $\begin{array}{l}1,02 \pm \\
0,02\end{array}$ & 1,93 & 372,44 & 8,66 & $\begin{array}{c}0,99 \pm \\
0,01\end{array}$ & 1,41 & 373,33 & 9,39 \\
\hline $\begin{array}{l}\text { Standard R1 } \\
\text { Padrão R1 }\end{array}$ & $\begin{array}{c}5,66 \pm \\
0,49\end{array}$ & 8,66 & 383,00 & 0 & $\begin{array}{c}4,49 \pm \\
0,50\end{array}$ & 11,09 & 383,11 & 0,087 \\
\hline $\begin{array}{l}\text { Standard R2 } \\
\text { Padrão R2 }\end{array}$ & $\begin{array}{c}5,14 \pm \\
0,51\end{array}$ & 9,99 & 384,00 & 0 & $\begin{array}{c}4,56 \pm \\
0,58\end{array}$ & 12,79 & 383,44 & 0,14 \\
\hline $\begin{array}{l}\text { Formulation F } \\
\text { R1 } \\
\text { Formulação F } \\
\text { R1 }\end{array}$ & $\begin{array}{c}5,66 \pm \\
0,49\end{array}$ & 17,39 & 384,44 & 0,14 & $\begin{array}{c}4,93 \pm \\
0,98\end{array}$ & 19,79 & 384,00 & 0 \\
\hline $\begin{array}{l}\text { Formulation F } \\
\text { R2 } \\
\text { Formulação F } \\
\text { R2 }\end{array}$ & $\begin{array}{c}4,67 \pm \\
0,81\end{array}$ & 8,53 & 384,78 & 0,11 & $\begin{array}{l}3,35 \pm \\
0,686\end{array}$ & 20,50 & 386,67 & 0 \\
\hline $\begin{array}{l}\text { Formulation } \\
\text { G R1 } \\
\text { Formulação G } \\
\text { R1 }\end{array}$ & $\begin{array}{c}4,20 \pm \\
0,36\end{array}$ & 16,02 & 384,11 & 0,08 & $\begin{array}{c}4,03 \pm \\
0,52\end{array}$ & 12,85 & 384,00 & 0,13 \\
\hline $\begin{array}{l}\text { Formulation } \\
\text { G R2 } \\
\text { Formulação G } \\
\text { R2 }\end{array}$ & $\begin{array}{c}3,82 \pm \\
0,61\end{array}$ & 23,49 & 383,89 & 0,06 & $\begin{array}{c}6,70 \pm \\
1,45\end{array}$ & 29,17 & 384,44 & 0 \\
\hline $\begin{array}{l}\text { Formulation } \\
\text { H R1 } \\
\text { Formulação H } \\
\text { R1 }\end{array}$ & $\begin{array}{c}4,64 \pm \\
0,50\end{array}$ & 36,20 & 385,00 & 0 & $\begin{array}{c}5,19 \pm \\
0,51\end{array}$ & 21,63 & 384,44 & 0,14 \\
\hline $\begin{array}{l}\text { Formulation } \\
\text { H R2 } \\
\text { Formulação H } \\
\text { R2 }\end{array}$ & $\begin{array}{c}4,34 \pm \\
1,57\end{array}$ & 22,93 & 384,33 & 0,13 & $\begin{array}{c}4,51 \\
\pm 1,49\end{array}$ & 33,22 & 384,33 & 0,19 \\
\hline
\end{tabular}


The Blank formulation showed lower values in the coefficient of variation (Table 8). The Standard formulation showed more variation, and formulations F, G and $\mathrm{H}$ showed even greater variation (particularly formulation $\mathrm{H}$ ). The H R1 and R2 samples (both measurements), F R1 and F R2 (both measurements) and G R2 (both measurements) showed high coefficients of variation, exceeding the $20 \%$ threshold used as the exclusion parameter for the measurements. When the coefficient of variation is higher than $20 \%$, the formulation was not adequately homogenized in the stand / plate used in the test, or the formulation itself was not homogeneous and stable.

The UVA efficacy was determined from the critical wavelength values (Table 8). The Blank formulation showed values from 372.33 to $393.44 \mathrm{~nm}$. The other formulations (Standard, F, G and H) presented critical wavelength values ranging from 383.00 to $386.67 \mathrm{~nm}$. These parameters, above $370 \mathrm{~nm}$, suggest that the formulation has the potential to achieve wide spectrum of absorption, as long as there is a substantial increase in SPF. The formulations with vegetable butters did not allow the expansion of the action spectrum and did not show statistically different results according to the Tukey method. According to this same methodology, the Blank and Standard formulations also showed statistically equal critical wavelength values.

\section{Discussão}

Foi possível desenvolver formulações fotoprotetoras contendo 2,0\% de Aristoflex ${ }^{\circledR}$ AVC e 5,0\% de triglicérides do ácido cáprico caprílico (GTCC). Não foram adicionados tensoativos convencionais à formulação. Isto foi possível devido à presença do Aristoflex ${ }^{\circledR} \mathrm{AVC}$. Analisando sua estrutura química, foi possível verificar que cada monômero deste polímero possui grupamentos $\mathrm{SO}^{3-} \mathrm{NH}^{4+}$ com característica polar. O restante da cadeia apresenta porções apolares. Sua incorporação na formulação foi realizada na fase oleosa. A aplicação do emoliente GTCC facilitou a dispersão dos ativos. A suspensão de dióxido de titânio já continha este emoliente entre seus componentes.

A escolha destes componentes para o desenvolvimento da formulação permitiu a obtenção de uma preparação com diversas características vantajosas. Entre elas, o seu sensorial agradável, não pegajoso (graxo), sensação de refrescância e boa espalhabilidade.

Os resultados de FPS foram obtidos por meio de análise em Labsphere ${ }^{\circledR}$ UV 2000S utilizando Transpore ${ }^{\circledR}$ (Tabela 8). Esta alternativa apresenta baixo custo, facilidade no manuseio e é adequada para amostras que não possuem óleo em excesso ou álcool como veículo.

Table 9/ Tabela 9 - Results of statistical analysis for SPF values by the Tukey method comparing the standard sample with the F, G and $\mathrm{H}$ samples.

Resultados da Análise estatística para valores de FPS pelo método de Tukey comparando amostra Padrão com as amostras F, G e H.

ns (not significant); CI (confidence interval)

ns (não significante); IC (Intervalo de confiança)

\begin{tabular}{|c|c|c|c|c|c|}
\hline \multirow{3}{*}{$\begin{array}{l}\text { Tukey's Multiple } \\
\text { Comparison Test }\end{array}$} & \multirow{3}{*}{$\begin{array}{c}\text { Meas. } \\
\text { Difference } \\
\text { Med. } \\
\text { Diferença }\end{array}$} & \multirow[b]{3}{*}{$\mathbf{Q}$} & \multicolumn{3}{|c|}{ Summary } \\
\hline & & & Significant/Significativo & 1 & 95\% CI difference/ \\
\hline & & & $\mathrm{P}<0.05$ & Resumo & IC de diferença \\
\hline $\begin{array}{l}\text { Standard vs F } \\
\text { Padrão vs F }\end{array}$ & $-0,8333$ & 3,503 & No/ Não & ns & -1.832 to 0.1655 \\
\hline $\begin{array}{l}\text { Standard vs G } \\
\text { Padrão vs G }\end{array}$ & $-0,7222$ & 3,036 & No/ Não & ns & -1.721 to 0.2766 \\
\hline $\begin{array}{c}\text { Standard vs H } \\
\text { Padrão vs H }\end{array}$ & $-0,8889$ & 3,737 & No/ Não & ns & -1.888 to 0.1100 \\
\hline
\end{tabular}


Table 10/ Tabela 10 - Results of statistical analysis for Lambda critical values by the Tukey method comparing standard sample with the F, G and $\mathrm{H}$ samples.

Resultados da Análise estatística para valores de Lambda crítico pelo método de Tukey comparando amostra Padrão com as amostras F, G e H.

ns (not significant); CI (confidence interval

ns (não significante); IC (Intervalo de confiança)

\begin{tabular}{ccccccc}
\hline & $\begin{array}{c}\text { Meas. } \\
\text { Difference } \\
\text { Tukey's Multiple } \\
\text { Comparison Test }\end{array}$ & $\begin{array}{c}\text { Med. } \\
\text { Diferença }\end{array}$ & $\mathbf{Q}$ & $\begin{array}{c}\text { Significant/Significativo } \\
\mathbf{P}<\mathbf{0 . 0 5}\end{array}$ & $\begin{array}{c}\text { Summary } \\
\text { Resumo }\end{array}$ & $\begin{array}{c}\text { 95\% CI } \\
\text { difference/ } \\
\text { IC de diferença }\end{array}$ \\
\hline $\begin{array}{c}\text { Standard vs F } \\
\text { Padrão vs F }\end{array}$ & 0,6792 & 1,713 & No/ Não & ns & -0.9859 to 2.344 \\
\hline $\begin{array}{c}\text { Standard vs G } \\
\text { Padrão vs G }\end{array}$ & 0,5483 & 1,383 & No/ Não & ns & -1.117 to 2.213 \\
\hline $\begin{array}{c}\text { Standard vs H } \\
\text { Padrão vs H }\end{array}$ & 0,0005150 & 0,001299 & No/ Não & & ns & -1.665 to 1.666 \\
\hline
\end{tabular}

Table 11/ Tabela 11 - Results of statistical analysis for critical Lambda values and SPF by the Tukey method comparing standard sample with the Blank sample.

Resultados da Análise estatística para valores de Lambda crítico e FPS pelo método de Tukey comparando amostra Padrão com a amostra Branco.

SPF (sun protection factor)

FPS (fator de proteção solar)

\begin{tabular}{|c|c|c|}
\hline $\begin{array}{l}\text { Blank X Standard } \\
\text { Branco X Padrão }\end{array}$ & $\begin{array}{l}\text { FPS } \\
\text { SPF }\end{array}$ & $\begin{array}{c}\text { Lamda crítico } \\
\text { Critical } \\
\text { Lambda }\end{array}$ \\
\hline $\mathrm{P}$ value/ Valor $\mathrm{P}$ & $\mathrm{P}<0.0001$ & 0.7107 \\
\hline $\mathrm{P}$ value / Valor P(summary/sumário) & $* * *$ & ns \\
\hline $\begin{array}{c}\text { Are the variances significantly different? } \\
\text { As variâncias são significativamente diferentes? }\end{array}$ & Yes / Sim & No/Não \\
\hline
\end{tabular}




\section{Conclusions}

It was possible to obtain photoprotective formulations using $2.0 \%$ of Aristoflex ${ }^{\circledR}$ and $5.0 \%$ of GTCC associated with titanium dioxide in suspension and bioactive cocoa, murumuru and cupuaçu butters. Formulations containing these butters showed no significantly higher SPF values when compared to the Standard sample. The use of only one physical sunscreen in the formulation was insufficient for higher protection. It was concluded, in accordance with the spectral profile and critical wavelength, that the action spectrum of the Blank, Standard, F, G and H formulations was approximately 290-385 nm.

Although the results do not indicate a possible increase in the amount of SPF or an expansion in the absorption spectrum, these study results indicate that the use of these butters in sunscreens may be beneficial due to their emollient and antioxidant properties (FRANQUILINO, 2006; ABURJAI, NATSHEH 2003; GASSER, et al, 2008;. SANTOS, et al, 2010).

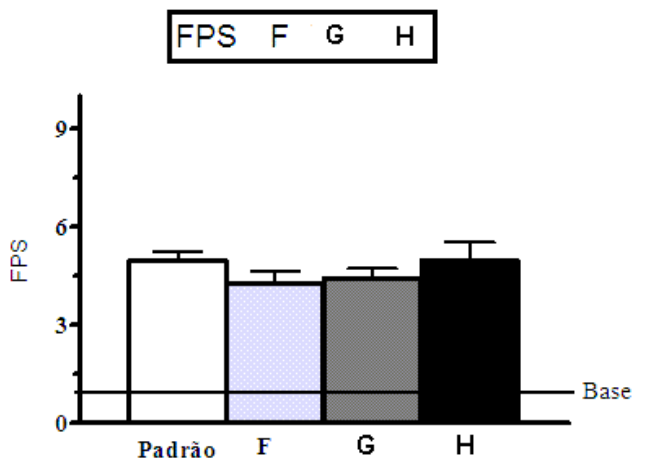

Formulations/Formulações

Figure 3/ Figura 3 - Graph of SPF values for Standard formulations F, G and $\mathrm{H}$. Gráfico de valores de FPS para as formulações Padrão, F, G e H.
Os valores de FPS para formulação Branco foram significativamente inferiores comparando-se com os valores das outras. As formulações $\mathrm{F}, \mathrm{G}$ e $\mathrm{H}$ apresentaram valores de FPS semelhantes aos apresentados pela formulação Padrão que continha apenas dióxido de titânio. Os valores de FPS nesses casos variou entre 4 e 5. Desse modo, as manteigas vegetais não apresentaram aumento do FPS das formulações, em suas concentrações utilizadas, de acordo com os valores obtidos por esta análise.

A formulação Branco apresentou menores valores no coeficiente de variação (Tabela 8). A formulação $\mathrm{Pa}$ drão apresentou maior variação e as formulações $F, G$ e $\mathrm{H}$ demonstraram ainda maior (especialmente a formulação H). As amostras H R1 e R2 (nas duas medições), F R1 e F R2 (nas duas medições) e G R2 (nas duas medições), apresentaram coeficientes de variação elevados, excedendo o limite de $20 \%$ utilizado como parâmetro de exclusão de medições. Quando o coeficiente de variação apresenta-se superior a $20 \%$, a formulação não foi adequadamente homogeneizada no suporte/ placa utilizada no teste ou a própria formulação não foi homogênea ou estável.

\section{Control FPS/ \\ Controle FPS}

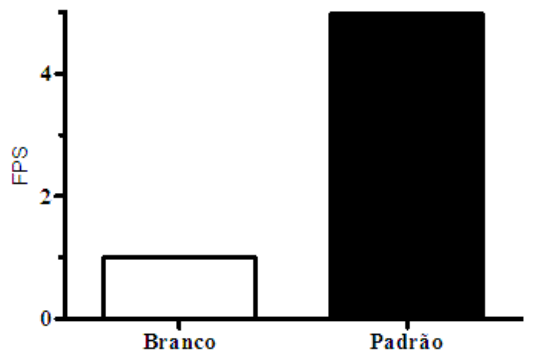

Formulations/Formulações

Figure 4/ Figura 4 - Graph of SPF values for Standard and Blank formulations.

Gráfico de valores de FPS para as formulações Padrão e Branco. 


\section{Conflict of Interests}

The authors declare that there are no financial and/or personal relationships that could be viewed as presenting a potential conflict of interests.

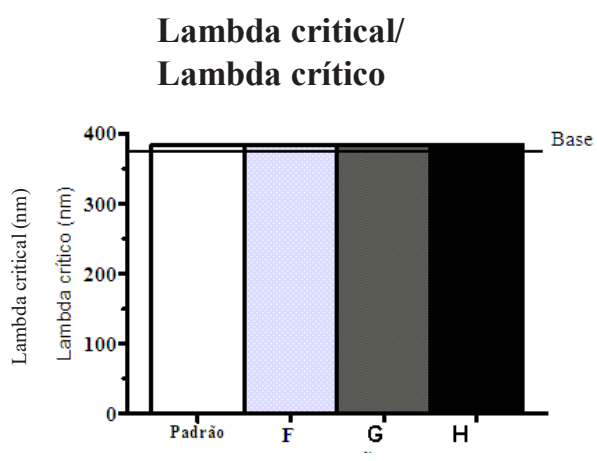

Formulations/Formulações

Figure 5/ Figura 5 - Lambda critical values for the Standard formulations F, G and $\mathrm{H}$.

Gráfico de valores de Lambda crítico para as formulações Padrão, F, G e H.

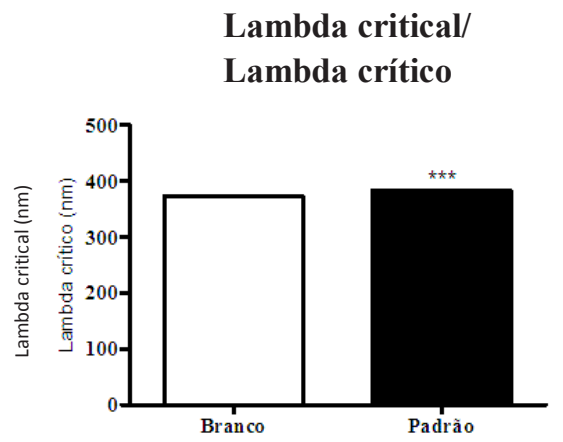

Formulations/Formulações

Figure 6/ Figura 6 - Lambda critical values for the Standard and blank formulations.

Gráfico de valores de Lambda crítico para as formulações Padrão e Branco.
A eficácia UVA foi avaliada a partir dos valores de comprimento de onda crítico obtidos. A Formulação Branco apresentou valores de 372,33 a 393,44 $\mathrm{nm}$. As demais (Padrão, F, G e H) apresentaram valores de comprimento de onda críticos variando de 383,00 a $386,67 \mathrm{~nm}$. Este parâmetro, acima de $370 \mathrm{~nm}$, sugere que a preparação possui potencial para atingir amplo espectro de absorção, desde que haja substancial elevação do FPS. As formulações com manteigas vegetais não possibilitaram a ampliação do espectro de ação e não apresentaram resultados estatisticamente diferentes de acordo com o método de Tukey. De acordo com este mesmo método, as formulações Branco e Padrão também apresentaram valores de comprimento de onda crítico estatisticamente iguais.

\section{Conclusões}

Foi possível obter formulações fotoprotetoras utilizando $2,0 \%$ de Aristoflex ${ }^{\circledR}$ e 5,0\% de GTCC associadas ao dióxido de titânio em suspensão e manteigas bioativas de cacau, murumuru e cupuaçu. As formulações contendo manteigas não apresentaram FPS significativamente superior ao da amostra Padrão. A utilização de apenas um filtro solar físico na formulação demonstrou-se insuficiente para proteção mais elevada.

Foi possível concluir, de acordo com o perfil espectral e comprimento de onda crítico, que o espectro de ação das formulações Branco, Padrão, F, G e H foi de aproximadamente 290 a $385 \mathrm{~nm}$.

Apesar dos resultados não indicarem um possível incremento no valor de FPS ou ampliação no espectro de absorção, foi permitido ressaltar que o uso dessas manteigas em protetores solares pode ser benéfico devido às suas características emolientes e antioxidantes (FRANQUILINO, 2006; ABURJAI, NATSHEH, 2003; GASSER, et al., 2008; SANTOS, et al., 2010).

\section{Conflito de Interesses}

Os autores declaram não existir qualquer relação pessoal ou financeira que possa ser entendida como representando um potencial conflito de interesses. 


\section{References/ referências}

(1) Guarantini T, Callejon DR, Pires DC, Lopes JNC, Lima LM, Neto DG, Sustovich C, Lopes NP. Fotoprotetores derivados de produtos naturais: perspectivas de mercado e interações entre o setor produtivo e centros de pesquisa. Química Nova 2009; 32(3): 717-21.

(2) Ichihashi M, Ueda M, Budiyanto A, Bito, T, Oka M, Fukunaga M, Tsuru K, Horikawa T. UV-induced skin damage. Toxicology 2003;189(1-2): 21-39.

(3) Matsumura Y, Ananthaswamy HN. Toxic effects of ultraviolet radiation on the skin. Toxicology and Applied Pharmacology 2005; 195:298- 308 .

(4) Scarlett WL. Ultraviolet radiation: sun exposure, tanning beds, and vitamin $\mathrm{D}$ levels. What you need to know and how to decrease the risk of skin cancer. The Journal of the American Osteopathic Association 2003;103(8):371- 5.

(5) Seité S, Fourtanier AM. The benefit of daily photoprotection. Journal of American Academy of Dermatology 2008; 58( 5): 160-1.

(6) Camacho F. Antiguos y nuevos aspectos de la fotoprotección. Revista Internacional de Dermatología y Dermocosmética Clínica 2001;4( 7): 441-7.

(7) Maier T, Korting HC. Sunscreens - Which and What for? Skin Pharmacol Physiol 2005;18:253-62.

(8) Baby AR, et al. UVA I-Protection effectiveness of bioactive compound and organic uv filters: an in vitro assessment. Química Nova 2009 ; 32(5):1321-3.

(9) Flor J, Davolos MR, Correa MA. Protetores Solares. Química Nova 2007;30(1):153-8.
(10) Serpone N, Dondi D, Albini A. Inorganic and organic UV filters: Their role and efficacy in sunscreens and suncare products. Inorganica Chimica Acta 2007 ;360: 794-802.

(11) Cook N, Freeman S. Report of 19 cases of photoallergic contact dermatitis to sunscreens seen at the Skin and Cancer Foundation. Australasian Journal of Dermatolog 2001;42( 4): 257-9.

(12) Butt ST, Christensen T. Toxicity and Phototoxicity of chemical sun filters. Radiation Protection Dosimetry 2000;91(1-3):283-6.

(13) Darvauy A, White AR, Rycroft RJ, Jones AB, Hawk JL, Mcfadden JP. Photoallergic contact dermatitis is uncommon. British Journal of Dermatology 2001 ;145: 597601.

(14) Aburjal T, Natsheh FM. Plants Used in Cosmetics. Phytotherapy Research 2003; 17:987-1000.

(15) Matsui MS, Hsia A, Miller JD, Hanneman K, Scull H, Cooper KD, Baron E. Non-Sunscreen Photoprotection: Antioxidants Add Value to a Sunscreen. Journal of Investigative Dermatology Symposium Proceedings 2009;14,: 56-9.

(16) Santos JS. Antioxidantes de Origem Vegetal em Cosméticos. Cosmetics and Toiletries (ed. Port.) 2010; 22(3): 46-54.

(17) Velasco MVR, Sarruf FD, Salgado-Santos IMN, Haroutiounian-Filho CA, Kaneko TM, Baby AR. Broad spectrum bioactive sunscreens. Int J Pharm 2008; 363( 1-2):50- 7.
(18) Zanatta CF, Mitjans M, Urgatondo V, Rocha-Filho PA, Vinardell MP. Photoprotective potential of emulsions formulated with Buriti oil (Mauritia flexuosa) against UV irradiation on keratinocytes and fibroblasts cell lines. Food Chem Toxicol 2010;48:70-5.

(19) Franquilino E. Ativos Amazônicos. Cosmetics and Toiletries (Ed Port) 2007 ; 18:18-53.

(20) Oliveira AC, Valentin IB, Goulart MOF, Silva CA, Bechara EJH, Trevisan MTS. Fontes vegetais naturais de Antioxidantes. Química Nova $2009 ; 32(3): 689-702$.

(21) Gasser P, Lati E, Peno-Mazzarino L, Bouzoud D, Allegaert L, Bernaert H. Cocoa polyphenols and their influence on parameters involved in ex vivo skin restructuring. Int J of Cosmetic Sci 2008 ;30: 339-345.

(22) Genovese MI, Lannes SC. Comparison of total phenolic content and antiradical capacity of powders and "chocolates" from cocoa and cupuassu. Ciência e Tecnologia dos Alimentos 2009;29( 4): 810-4.

(23) Silva EPO Castro L, Biaggio RM, Beltrame Jr M. Estudo das características físicoquímicas e classificação de fito-ingredientes na espécie Astrocaryum murumuru (murumuru). XII Encontro Latino Americano de Iniciação Científica e VIII Encontro Latino Americano de Pós-Graduação 2010:1-3.

(24) Dominique M. How to Measure UVA Protection Afforded by Sunscreen Products. Expert Review of Dermatology 2008; 3(3): 307-13. 